\title{
The chance case history of an unusual disease: mesenteric panniculitis
}

\author{
Salvatore Santo Signorelli $^{1} \cdot$ Valerio Fiore $^{1} \cdot$ Marco Mangiafico $^{1} \cdot$ Caltabiano Giuseppe $^{2}$
}

Received: 7 April 2015/Accepted: 25 April 2015/Published online: 13 May 2015

(C) SIMI 2015

Mesenteric panniculitis was first described by M. Jura in 1927 [1], and is characterized by large mesenteric infiltrations of plasma cells and polymorphonucleate cells. This disease is also known as mesenteric lipogranulomatosis, retroperitoneal xanthogranulomatosis, mesenteric lipodystrophia and mesenteric panniculitis [2-4]. Acute, sub-acute or recurrent abdominal pain occurs in occlusive or subocclusive cases; fever, weight loss and chronic anaemia may be described by patients. Imaging radiology examinations (CT scan, MRI) are now considered very useful in diagnosing the disease, although biopsy also plays a crucial role in diagnostics (Figs. 1, 2).

An 84-year-old man suffering from long-term chronic lymphatic leukaemia recently experienced recurrent acute pain in the middle and lower abdomen. Clinical examination revealed no diagnostic signals (abdominal quadrants were easily palpable, both Murphy's and Mc Burney's test were negative, there were no clinical signs of acute renal pain). The patient's abdomen was examined by ultrasound, which showed only biliary microlithiasis. A CT scan of the upper abdomen was clear with non-homogeneous mesenteric adiposity plus many intra-parenchyma inclusions (lymph nodes). The CT scan clearly diagnosed mesenteric panniculitis. The CT images also showed a close relationship between this high adiposity with the part of the bowel that was consistent with the recurrent episodes of pain. The CT scan also showed a large aortic dilatation

Salvatore Santo Signorelli

ssignore@unict.it

1 Medical Angiology Unit, Department of Medical and Experimental Medicine, Garibaldi Hospital, University of Catania, Catania, Italy

2 Division of Radiology, Garibaldi Hospital, Catania, Italy with thrombotic occlusion (aortic aneurysm with thrombus).

The patient received a low-dosage (methylprednisolone $25 \mathrm{mg}$ daily) parenteral steroid therapy suggested by the haematologist to treat both the chronic haematological disease, and the unknown aortic dilatation was diagnosed and confirmed by non-invasive imaging techniques (echography, CT scan). The abdominal pain was relieved quickly, and the patient was surgically evaluated for aortic dilatation. The patient was sent to vascular surgery and treated for the aortic aneurysm.

The patient knew nothing about gallstones. Moreover, the abdominal echography showed that the inner and outer liver biliary tracts were not dilated and that the gallbladder shape, size and walls were normal and that any gallstones were very small. So, the biliary lithiasis treatment was limited to treating the pain.

The patient was recommended to undergo vascular surgery and received vascular prostheses.

Mesenteric panniculitis is a rare disease associated with auto-immune disease (e.g. Sjogren, rheumatoid arthritis, Riedel's thyroiditis, haemolytic anaemia) or with infectious disease (Tuberculosis) or with gastrointestinal disease (e.g. liver cirrhosis, pancreatic disease, retroperitoneal fibrosis) or with vascular diseases (e.g. vasculitides, mesenteric thrombosis, coronary artery disease, aortic abdominal aneurysm). Mesenteric panniculitis is often correlated with different gastric and bowel neoplasms (e.g. colorectal, lung, gastric, breast and renal cancer) or associated with skin cancer (melanoma) or thoracic disease (e.g. mesothelioma, carcinoid tumour). Interestingly, mesenteric panniculitis is also associated with haematological malignancies (e.g. non-Hodgkin lymphoma, Hodgkin's disease and chronic lymphatic leukaemia). In this case, we believe that mesenteric disease is a concomitant of the chronic 


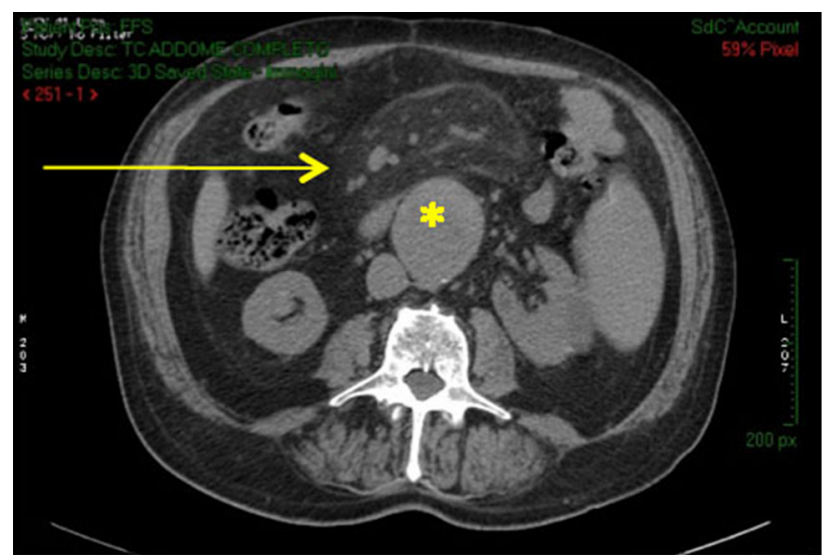

Fig. 1 CT scan shows mesenteric panniculitis (close to 4th portion of duodenum and to 1 st jejunum tract). Asterisk abdominal aortic aneurysm

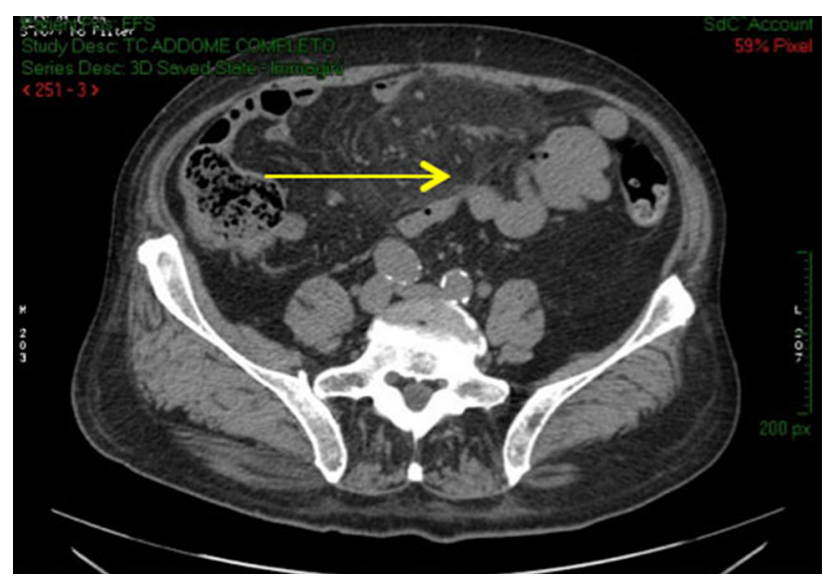

Fig. 2 CT scan shows that mesenteric panniculitis is close to ileal tract

haematological disease long suffered (more than 10 years) by the patient. This opinion is supported first by the patient's recent medical history, and also by the lack of any other clinical or laboratory signs leading to other diagnoses. Finally, we want to highlight the positive results produced by the steroid therapy.

We have highlighted this unusual disease to draw the attention of physicians not only to a rare clinical case, but also to avoiding the aggressive surgical option suggested by the clinical suggestion of acute intestinal obstruction. In fact, medical history and clinical normalization in due time by anti-phlogistic drugs must be considered as crucial issues. We also want to draw attention to the efficacy of noninvasive tests (echography and CT scan) in diagnosing this particularly unusual disease.

Conflict of interest The authors declare that they have no conflict of interest.

Statement of human and animal rights All procedures performed in studies involving human participants were in accordance with the ethical standards of the institutional and/or national research committee and with the 1964 Helsinki declaration and its later amendments or comparable ethical standards. This article does not contain any studies with human and animals performed by any of the authors.

Informed consent Informed consent was obtained from all individual participants included in the study.

\section{References}

1. Jura V (1927) Mesenterite retrattile-caso clinico: risultati sperimentali, rilievi patogenetici, considerazoni cliniche. Policlinico (sez. Chir) 34: 535-556, 566-599

2. Horton KM, Lawler LP, Fishman EK (2003) Findings in sclerosing mesenteritis (Panniculitis): spectrum of disease. Radiographica 23(6):1561-1567

3. Vettoretto N, Diana DR, Poiatti R, Matteucci A, Chioda C, Giovanetti M (2007) Occasional finding of mesenteric lipodystrophy during laparoscopy: a difficult diagnosis. World J Gastroenterol 13(40):5394-5396

4. Hussein MRA, Abdelwhewd SR (2015) Mesenteric panniculitis: an update. Exp Rev Gastroenterol Hepatol 9(11):67-78 